\title{
Detection of Information Hiding by Modulating Intra Prediction Modes in H.264/AVC
}

\author{
Songbin Li \\ Haikou Laboratory, Institute of Acoustics \\ Chinese Academy of Sciences \\ Haikou, China \\ e-mail:lisongbin_work@126.com \\ Peng Liu, Qiongxing Dai \\ Haikou Laboratory, Institute of Acoustics \\ Chinese Academy of Sciences \\ Haikou, China
}

\author{
Xiuhua Ma \\ College of Information Science and Technology \\ Hainan University \\ Haikou, China \\ e-mail:lingmaliao@sina.com \\ Haojiang Deng \\ National Network New Media Engineering Research \\ Center \\ Beijing, China
}

\begin{abstract}
In the intra-frame coding of H.264/AVC, information hiding can be implemented by modulating the prediction modes of $4 \times 4$ luminance blocks. Since this kind of method has characteristics of high speed, good concealment and so on, it has become a great security threat. Therefore, it is necessary to study the steganalysis method. In this paper, we first analyzed the changes of remarkable characteristics in intra-frame coding caused by modulating intra prediction modes for information hiding. We found that the correlation among the prediction modes in different $4 \times 4$ luminance blocks, belonging to an intra-frame coding macroblock, was changed. According to this, we have designed statistical models to make quantitative extraction of these correlation characteristics. And a steganography detector was constructed based on the support vector machine. The experimental results show that the steganography detector constructed in this paper can achieve a detection accuracy of more than $90 \%$ when the embedding rate is larger than $25 \%$.
\end{abstract}

Keywords-information hiding; steganalysis; H.264/AVC; intra prediction; prediction modes modulating

\section{INTRODUCTION}

H.264/AVC is a new generation of international video coding standard. It has been approved by ITU-T as Recommendation H.264 and by ISO/IEC as International Standard MPEG-4 part 10 Advanced Video Coding (AVC). Since it has been widely used, the H.264/AVC video streams have become a potential and much threatening information hiding carrier. If it is used to transmit covert communication, national communication regulation will be faced with a great threat. Therefore, it is necessary to study the detecting algorithm of information hiding.

The existing information hiding methods for H.264/AVC are mainly as follows: 1 ) the method based on quantized integer transform coefficients [1-3]; 2) the method based on motion vector [4-6]; 3) the method based on modulating intra prediction modes [7-9]. Since the third method is implemented by comparing and assigning operations on the basis of original coding, it does not increase much computation. Therefore, it has characters of low complexity and high speed, which makes it very suitable for information hiding in H.264/AVC. Reference [7] is the previous work of information hiding based on modulating prediction modes. Some later methods can be regarded as the improvement and development of it. So we take [7] as an example to describe the detection method of information hiding based on modulating prediction modes.

At present, most of the video stego-detecting algorithms are using the method of pattern recognition [10-12]. The key point of this method is to find out the characteristics of steganography sensitivity. Therefore, for [7] the difficulty of stego-detecting is to search and determine the remarkable characteristics. It is caused by modulating the I4 blocks' prediction modes in the H.264/AVC coding for steganography. Using the method above, we can detect whether the video is concealed with secret information.

\section{THE CHANGES OF REMARKABLE CHARACTERISTICS AFTER STEGANOGRAPHY}

\section{A. Intra prediction of the H.264/AVC}

In intra mode, a prediction block $P$ is formed based on previously encoded and reconstructed blocks and is subtracted from the current block prior to encoding. For the luminance samples, $P$ is formed for each $4 \times 4$ block or for a $16 \times 16$ macroblock. There are a total of nine optional prediction modes for each $4 \times 4$ luminance block, four modes for a $16 \times 16$ luminance block and four modes for the chrominance components. The encoder typically selects the prediction mode for each block that minimizes the difference between $P$ and the block to be encoded.

\section{B. The prediction modes of $4 \times 4$ luminance blocks}

Figure 1 a shows a $4 \times 4$ luminance block that is required to be predicted. The samples above and to the left (labeled $A-M$ in Figure 1a) have previously been encoded and reconstructed and are therefore available in the encoder and decoder to form a prediction reference. The samples 
$a, b, c, \ldots, p$ of the prediction block $P$ (Figure $1 \mathrm{a}$ ) are calculated based on the samples $A-M$ as follows. Mode 2 (DC prediction) is modified depending on which samples $A-M$ have previously been coded; each of the other only be used if all of the required prediction samples are available.

The arrows in Figure $1 \mathrm{~b}$ indicate the direction of prediction in each mode. For modes 3-8, the predicted samples are formed from a weighted average of the prediction samples $A-M$. For example, if mode 4 is selected, the top-right sample of $P$ (labeled "d" in Figure 1a) is predicted by:

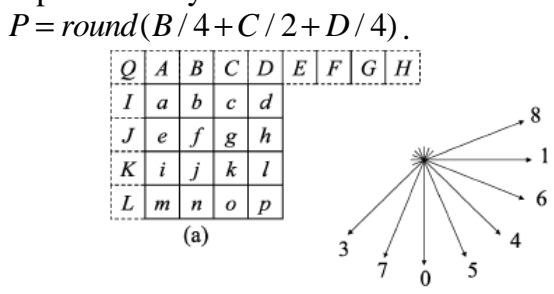

(b)

Figure 1. a) I4MB prediction coding is conducted for samples a-p of a block using samples A-Q; b) Eight “prediction directions” for I4MB prediction

\section{Analysis of mode correlation}

Information hiding in [7] is implemented by using the H.264/AVC's specific intra prediction modes. Since after steganography, only the prediction mode is changed. If we can give a quantitative analysis model to measure this change effectively, the information hiding can be detected. The occurrence frequency of different prediction modes and the correlation among them may be changed when their prediction modes are changed. The occurrence frequency of prediction modes is related to the image content. For example, if the image's texture trend is horizontal, the occurrence frequency of horizontal prediction mode will be high. Therefore, the occurrence frequency of prediction modes can not be used as a base of steganography detection. However, the correlation among prediction modes is determined by the image's local correlation and it is independent on the image content. Hereby, this paper tries to model the correlation among prediction modes to detect the steganography.

\section{A QUANTITATIVE STATISTICS MODEL FOR THE}

CORRELATION OF I4 BLOCKS' INTRA PREDICTION MODES

The information hiding method based on modulating prediction modes can affect the correlation among neighbor I4 blocks' prediction modes. We will give the quantitative statistics method of the characteristics in this section.

In order to quantitatively analyze the correlation among the neighbor I4 blocks, this paper assumes that a $4 \times 4$ luminance block is as shown in the Figure 2. And the prediction mode of I4 block $a_{i, j}(i, j \in[1,4])$ is $p_{i, j} \in[0,8]$. An I4 block may affect the surrounding I4 blocks' prediction modes from multiple directions. Taking an I4 block $a_{i, j}$ as an example, it may affect the prediction modes of its horizontal neighbor blocks $a_{i, j-1}$ and $a_{i, j+1}$. What is mo-re, it may also affect the prediction modes of its vertical neighbor blocks $a_{i-1, j}$ and $a_{i+1, j}$, etc. In theory, the I4 block $a_{i, j}$ can affect the surrounding I4 blocks' prediction modes from its eight prediction directions which are defined in the intra-frame coding of H.264/AVC. However, to simplify the calculation, we research the correlation among the I4 blocks' prediction modes only in the following directions: (1) horizontal; (2) vertical; (3) diagonal; (4) back diagonal. The equation is as follows:

$$
\left\{\begin{array}{l}
a_{i, j} \rightarrow a_{i, j+1} \\
a_{i, j} \rightarrow a_{i+1, j} \\
a_{i, j} \rightarrow a_{i+1, j+1} \\
a_{i, j} \rightarrow a_{i+1, j-1}
\end{array}\right.
$$

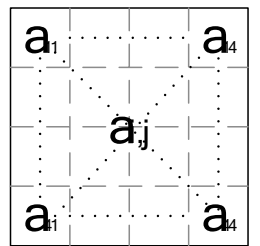

Figure 2. Neighbor I4 blocks

Based on the four directions mentioned above, we can obtain the four directions' sequence respectively, where the horizontal direction sequence is:

$$
S_{1}=a_{11} a_{12} a_{13} a_{14} a_{21} a_{22} a_{23} a_{24} a_{31} a_{32} a_{33} a_{34} a_{41} a_{42} a_{43} a_{44} \text {; }
$$

The vertical direction sequence is:

$$
S_{2}=a_{11} a_{21} a_{31} a_{41} a_{12} a_{22} a_{32} a_{42} a_{13} a_{23} a_{33} a_{43} a_{14} a_{24} a_{34} a_{44} \text {; }
$$

The diagonal direction sequence is:

$S_{3}=a_{12} a_{21} a_{13} a_{22} a_{31} a_{14} a_{23} a_{32} a_{41} a_{24} a_{33} a_{42} a_{34} a_{43}$;

The back diagonal direction sequence is:

$S_{4}=a_{13} a_{24} a_{12} a_{23} a_{34} a_{11} a_{22} a_{33} a_{44} a_{21} a_{32} a_{43} a_{31} a_{42}$.

According to above, the value of the elements $a_{i, j}$ in the sequence $S_{i}$ is $p_{i, j}$. If we take $a_{i, j}$ as a variable, the sequence $S_{i}$ can be modeled as a random process: $\left\{S_{p}\left(t_{n}\right), t_{n} \in T\right\}$, where $T=[0,1,2, \ldots]$. In order to analyze the correlation among the prediction modes in sequence $S_{p}\left(t_{n}\right)$, the random process corresponding to the sequence can be regarded as a Markov Process. The current state of the sequence is determined by its past $h$ states. The equation is as follows:

$$
\operatorname{Pr}\left(S_{p}\left(t_{h+1}\right)=p_{j} \mid S_{p}\left(t_{h}\right)=p_{i_{h}}, S_{p}\left(t_{h-1}\right)=p_{i_{h-1}}, \ldots, S_{p}\left(t_{1}\right)=p_{i_{1}}\right)
$$

Where $S_{p}\left(t_{n}\right)$ is the state when the random variable is at the time of the $t_{h}$, and its value is $p_{i} \cdot p_{i}$ is the I4 blocks' nine prediction modes in the intra-frame coding. $p_{i_{h}}$ is the same as the $p_{i}$ and it means at the time $h$ the state is $p_{i}$. Since computing the equation directly is difficult, we assume the appearance of the current state is only related to 
its first past state. Therefore, equation (2) can be simplified as (3).

$$
\operatorname{Pr}\left(S_{p}\left(t_{h+1}\right)=p_{j} \mid S_{p}\left(t_{h}\right)=p_{i_{h}}\right)
$$

Hereby, the correlation among the prediction modes can be measured by the state transition matrix $P\left(t_{h}\right)$ of the onestep Markov Process. If the number of the states is $N$, the transition probability matrix of the one-step model is $N \times N$. Obviously, the state transition matrix can quantize the correlation among the I4 blocks' prediction modes well.

\section{THE PROCESS OF STEGANALYSIS}

We select the video frames of H.264 intra-frame coding as the test target. The steganalysis in this paper is intended to judge whether the video frames exist the steganography based on modulating prediction modes. The judgment result is only "yes" (we call "stego" type in this paper) or "no" (we call "cover" type in this paper). Therefore, the essence of the stego-detecting process is to classify. It mainly includes two steps: firstly, extract the feature vectors of the intra frames; secondly, use the feature vectors to train the classifier and then begin to classify. This paper constructs feature vectors based on correlation among prediction modes' statistical characteristics which are introduced in section 3. We study the statistical correlation among the prediction modes in four directions: horizontal, vertical, diagonal and back diagonal. Then we can obtain the predict-ion modes' transition matrix $P^{n}(n=1,2,3,4)$ in these four directions. We construct the feature vector $F=\left(P_{1,1}^{1}, P_{1,2}^{1}, \ldots, P_{9,9}^{1}, P_{1,1}^{2}, P_{1,2}^{2}, \ldots, P_{9,9}^{2}, P_{1,1}^{3}, P_{1,2}^{3}, \ldots, P_{9,9}^{3}, P_{1,1}^{4}, P_{1,2}^{4}, \ldots, P_{9,9}^{4}\right)$ based on $P^{n}$, where the $P_{i, j}^{n}(i, j \in\{1,2, \ldots, 9\})$ is the element of the transition matrix $P^{n}$. We call feature vector $F$ as the feature vector of prediction mode correlation (PMC). The SVM (Support Vector Machine) is used as the classifier, and the process of stego-detecting is shown in Figure 3.

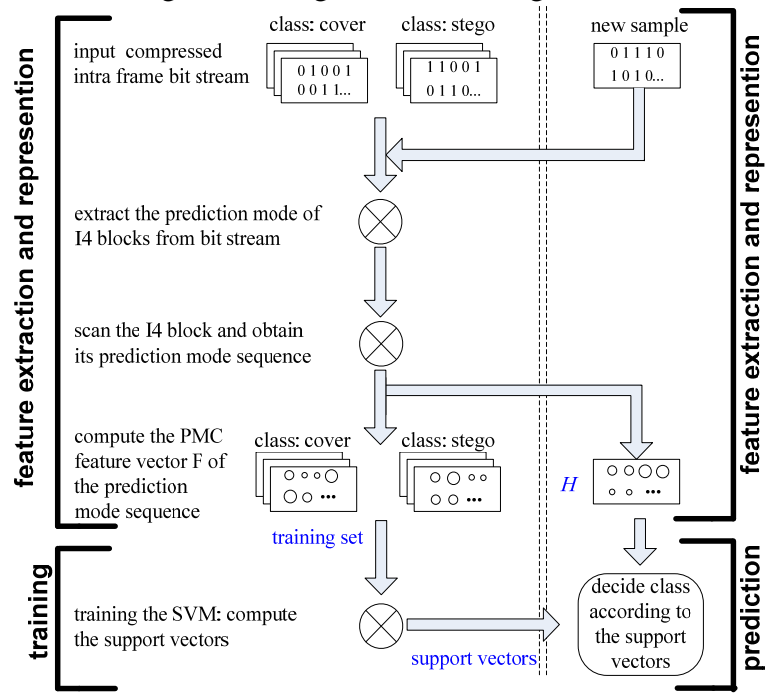

Figure 3. The stego-detecting process of intra-frame coding based on SVM.

\section{EXPERIMENT AND RESULT ANALYSIS}

For the test video sequence $V$, the experimental process is as follows: 1) Every video frame in the $V$ is coded as intra-frame using H.264 reference encoder JM11. Then we can obtain the I4 blocks' prediction mode information of every video frame; 2) Using the information hiding method given in [7] and based on different templates, every video frame in $V$ is coded as intra-frame once again. Then after that, we can obtain the I4 blocks' prediction mode information which is after steganography; 3) Assuming the number of the video frames in test video sequence $V$ is $L$. Then the number of the "cover" frames and the corresponding "stego" frames is also $L$. Take $75 \%$ of them as the training set and the rest as test set; 4) We extract the PMC characteristic from the samples in the training set and then train the SVM classifier; 5) Extract the PMC characteristic from every sample in the training set and begin to classify based on the SVM classifier in step 3. Then we record the detection accuracy of all the samples in the test set.

In order to prove the good universality of our method, we have experimented on many video sequences. The selected test video sequences in this paper are as follows: Claire (including 494 frames), Coastguard (including 300 frames), Container (including 300 frames), Foreman (including 300 frames), Miss America (including 150 frames), Mobile (including 300 frames), Mother and Daughter (including 300 frames), Salesman (including 449 frames) and Suzie (including 150 frames). In this paper, we first use the steps introduced above to record the detection accuracy of these sequences. Then we record the stegodetecting results of the hybrid sequence (called "hybrid", including 2743 frames), which is composed of all video frames in the nine video sequences. The results are shown in Table 1.

As is shown in Table 1:1) when the embedding rate is $12.5 \%$, the detection accuracies' mean value of all the test sequences are attaining to $91.26 \%$; 2) when the embedding rate is $25 \%$, the detection accuracies of all the test sequences are above $90 \%$. Except the hybrid sequence, there are six sequences' detection accuracies achieved 100\% already. The other three sequences' detection accuracies are all above $98.6 \%$; 3) when the embedding rate is $50 \%$, except the hybrid sequence, the rest sequences' detection accuracies are all attaining to $100 \%$; 4) Even though the embedding rate is low to $6.25 \%$, the detection accuracies' mean value are attaining to $77.74 \%$ using the stegodetecting method proposed in this paper. In a word, the stego-detecting algorithm proposed in this paper can detect not only the information hiding method given in [7] but also the methods with lower embedding rate. 
TABLE I. STEGO-DETECTION RESULTS

\begin{tabular}{cccccccc}
\hline \hline & $\mathbf{6 . 2 5 \%}$ & $\mathbf{1 2 . 5 \%}$ & $\mathbf{2 5 \%}$ & $\mathbf{5 0 \%}$ & $\mathbf{7 5 \%}$ & $\mathbf{1 0 0 \%}$ \\
\hline claire & $89.02 \%$ & $96.75 \%$ & $100 \%$ & $100 \%$ & $100 \%$ & $100 \%$ \\
coastguard & $84.67 \%$ & $96 \%$ & $100 \%$ & $100 \%$ & $100 \%$ & $100 \%$ \\
container & $94.67 \%$ & $100 \%$ & $100 \%$ & $100 \%$ & $100 \%$ & $100 \%$ \\
foreman & $62 \%$ & $84.67 \%$ & $98.67 \%$ & $100 \%$ & $100 \%$ & $100 \%$ \\
miss-america & $72.97 \%$ & $87.84 \%$ & $98.65 \%$ & $100 \%$ & $100 \%$ & $100 \%$ \\
mobile & $68.67 \%$ & $89.33 \%$ & $100 \%$ & $100 \%$ & $100 \%$ & $100 \%$ \\
Mother-daughter & $88 \%$ & $100 \%$ & $100 \%$ & $100 \%$ & $100 \%$ & $100 \%$ \\
salesman & $85.61 \%$ & $99.24 \%$ & $100 \%$ & $100 \%$ & $100 \%$ & $100 \%$ \\
suzie & $68.92 \%$ & $81.08 \%$ & $98.65 \%$ & $100 \%$ & $100 \%$ & $100 \%$ \\
hybrid & $62.85 \%$ & $77.73 \%$ & $90.07 \%$ & $98.47 \%$ & $99.35 \%$ & $99.12 \%$ \\
\hline \hline
\end{tabular}

\section{CONCLUTION}

In this paper, we have studied the stego-detecting algorithm based on modulating the intra prediction modes of I4 blocks. We found that it will result in a significantly changing in the correlation among different modes. According to this, we have constructed a stego-detecting algorithm. At last, we have experimented on a large number of video samples. The test results show that our method is effective.

\section{ACKNOWLEDGMENT}

This work is supported in part by National High Technology Research and Development Program of China under grant 011AA01A102 and in part by Strategic Priority Research Program of the Chinese Academy of Sciences under grant XDA06010302.

\section{REFERENCES}

[1] Jean-Marc Thiesse, Joël Jung, Marc Antonini, "Data Hiding of Intra Prediction Information in Chroma Samples for Video Compression,” Proceedings of the 2010 IEEE 17th International Conference on Image Processing, Sep.2010, pp.2861-2864, doi:10.1109/ICIP.2010.5652005.

[2] Ma Xiaojing, “A Study on the method of H.264/AVC video information hiding ," Ph.D. Thesis, Huazhong University of Science and Technology, Wu Han, 2010.

[3] Liwei Zhang, Yuesheng Zhu, Lai-Man Po, “A Novel Watermarking Scheme with Compensation in Bit-Stream Domain for h.264/AVC,” IEEE International Conference on Acoustics, Speech, and Signal Processing,Mar.2010, pp.1758-1761,doi: 10.1109/ICIP.2010.5652005.

[4] Jean-Marc Thiesse, Joël Jung, Marc Antonini, "Data Hiding of Motion Information in Chroma and Luma Samples for Video Compression," 2010 IEEE International Workshop on MultimediaProcessing, Oct. 2010, pp.217-221, doi:10.1109/MMSP 2010.5662022
[5] Hao Bin, Zhao Li-Yi, Zhong Wei-Dong, "A Novel Steganography Algorithm Based on Motion Vector and Matrix Encoding," 2011 IEEE 3th International Conference on Communication Software and Networks,May.2011, pp.406-409, doi: 10.1109/ICCSN.2011.6013622.

[6] Wang Jue, Zhang Min-qing, Sun Juan-li, "Video Steganography Using Motion Vector Components,” 2011 IEEE 3th International Conference on Communication Software and Networks, May. 2011, pp.500-503, doi: 10.1109/ICCSN.2011.6013642.

[7] Hu Yang, Zhang Chun-tian, Su Yu-ting, "Information Hiding for H.264/AVC," Acta Electronica Sinica, Vol.36, Apr. 2008, pp.690-694. doi:10.1109/ICME.2007.4284879.

[8] Wang Rang-ding, Zhu Hong-liu, Xu Da-wen, "Information hiding algorithm for H.264/AVC based on encoding mode," Opto-Electronic Engineering,Vol.37, May. 2010, pp.144-150. doi:10.3969/j.issn.1003501X.2010.05.026.

[9] Xu Da-wen, Wang Rang-ding, Wang Ji-cheng, "Prediction mode modulated data-hiding algorithm for H.264/AVC," Journal of Real-Time Image Processing, Vol.7, Nov. 2012, pp.205-214. doi:10.1007/s11554-0100175-4.

[10] Su Yu, Hui-Xiang Xu, Zhi-Hong Yang, Zhi-Chao Li, “A Novel Steganalysis Scheme of Digital Video," 2010 International Conference on Multimedia Information Networking and Security, Nov.2010, pp.952956,doi: 10.1109/MINES.2010.203.

[11] K. Kancherla, S. Mukkamala, "Video Steganalysis Using Motion Estimation,” 2009 International Joint Conference on Neural Networks, Jun.2009, pp.1510-1515, doi: 10.1109/IJCNN.2009.5179032.

[12] Chengqian Zhang, Yuting Su, Chuntian Zhang, “A New Video Steganalysis Algorithm Against Motion Vector Steganography,” 2008 4th International Conference on Wireless Communications, Networking and Mobile Computing, Oct.2008, pp.1-4, doi: 10.1109/WiCom.2008.781. 